\title{
Nutzen und Kosten der
}

\section{derzeitigen Regulierung \\ des Apothekenmarktes in}

Deutschland

Die vorliegende Untersuchung beschäftigt sich mit der ökonomischen Analyse von Nutzen und Kosten der derzeitigen staatlichen Regulierung des deutschen Apothekenmarktes. Es wird gezeigt, dass eine partielle Deregulierung in der Lage wäre, Effizienz und Qualität der Arzneimitteldistribution zu steigern. Insbesondere Fremd- und Mehrbesitzverbot sind für die Gesellschaft dabei mit hohen Kosten verbunden. Obgleich nicht alle Regulierungsmaßnahmen abzulehnen sind, wären zusätzliche oder sogar stärkere positive Effekte möglich, wenn zudem ein größeres Maß an Freiraum beim Versandhandel und der Preissetzung sowie partielle Lockerungen der Regulierung im Bereich des Geschäftsbetriebs und des Sortiments realisiert würden. Langfristige Folge der Versuchs- und Entdeckungsprozesse verschiedener Konzepte vertikaler und horizontaler Kooperation könnte ein wohlfahrtssteigerndes Nebeneinander von inhabergeführten Apotheken und solchen im Eigentum von Kapitalgesellschaften sein, wobei insgesamt eine verbesserte Erfüllung der gesellschaftlichen Präferenzen zu erwarten ist.

\section{Johann-Matthias Graf v. d. Schulenburg, Jan-Marc Hodek}

\section{Einleitung}

Alle Märkte unterliegen in unserer sozialen Marktwirtschaft einer generellen Regulierung. Dazu gehören die Regeln des Zivil- und Handelsrechts genauso wie die des Arbeits- und Wettbewerbsrechts. Die Begründungen für diese generellen Marktregulierungen sind vielfältig und zum Teil nicht unumstritten. Allerdings kann man sie in zwei Argumenten zusammenfassen: Um zu funktionieren braucht die Marktwirtschaft erstens Vertragstreue und ausreichend intensiven Wettbewerb zwischen den Anbietern sowie zweitens Verbraucherschutz und Arbeitnehmerrechte, welche in einer sozialen Marktwirtschaft hohe Priorität genießen.

Dazu treten für ganz bestimmte Märkte zusätzliche z.T. sehr umfangreiche staatliche Regulierungsrahmen. Zu

Prof. Dr. Johann-Matthias Graf v. d. Schulenburg, Leibniz Universität Hannover, Institut für Versicherungsbetriebslehre - Forschungsstelle Gesundheitsökonomie Dipl.-Ök. Jan-Marc Hodek,

Universität Bielefeld, Fakultät für Gesundheitswissenschaften, AG5 - Gesundheitsökonomie und Gesundheitsmanagement nennen ist die Sozialversicherung, das Banken- und Versicherungswesen, der Energiesektor, das Telekommunikationswesen oder die Energiewirtschaft. Zu diesen, ebenfalls nicht unumstrittenen spezifischen Marktregulierungen werden Begründungen geliefert, die in drei Gruppen zusammengefasst werden können: Soziale Fürsorge, Internalisierung externer Effekte und besondere Erfordernisse zur Sicherstellung des Verbraucherschutzes. ${ }^{1}$ Es gibt jedoch nur wenige Märkte, auf denen der Regulierungsrahmen so weitreichend ist wie auf dem Apothekenmarkt, da hier neben die Preis- und Geschäftsregulierungen eine Besitzregulierung tritt. Der Gesetzgeber legt fest, wer eine Apotheke besitzen und betreiben darf. Eben diese Regelungen standen und stehen seit Jahren in der ökonomischen, politischen und auch rechtlichen Diskussion. Obgleich beispielsweise die Wirtschaftsweisen des Sachverständigenrates (und auch die des Sachverständigenrates Gesundheit) seit Jahren für eine Liberalisierung der deutschen Regelungen zum Fremd- und Mehrbesitzverbot eintreten, folgte der Europäische Gerichtshof in seinem Urteil von Mitte Mai 2009 der Empfehlung des Generalanwaltes und bestätigte die aktuellen Bestimmungen als zielführend, angemessen und damit als gerechtfertigte Einschränkung der Niederlassungsfreiheit. Welche der beiden Seiten - zumindest 


\section{THEMA}

aus ökonomischer Perspektive - über die überzeugenderen Argumente verfügt, soll Gegenstand der vorliegenden Untersuchung sein. Diese betrachtet die Regulierung des Apothekenwesens aus ökonomischer Sicht und versucht zu bewerten, welche ökonomischen Effekte von verschiedenen Deregulierungsmaßnahmen ausgelöst würden. Neben einer regulierungstheoretischen Analyse der Arzneimitteldistribution wird aufgezeigt, welche Nutzen und Kosten von den einzelnen Maßnahmen ausgehen.

Dabei ist zu beachten, dass weder die Feststellung, die derzeitige Arzneimittelversorgung der Bevölkerung in Deutschland sei zufriedenstellend, eine Begründung für die Marktregulierungen ist, noch dass bei jedem (tatsächlichen oder vermeintlichen) Missstand in unserem Lande der Staat aufgerufen ist, regulierend einzugreifen. Regulierung stellt immer eine Einschränkung von Handlungsfreiheit der Konsumenten und/oder Anbieter dar. Deshalb muss jede Regulierung und die mit ihr verbundene Einschränkung der Freiheit sorgsam begründet und natürlich zur Zielerreichung geeignet sein.

Im Folgenden soll der Fragestellung nachgegangen werden, ob die größtenteils historisch gewachsenen und kürzlich durch das EuGH-Urteil bestätigten Regulierungsmaßnahmen für die Zielerreichung tatsächlich adäquat sind oder ob ein Fall von Fehlregulierung vorliegt. Ebenso sollen die (wohlfahrtstheoretischen) Folgen einer Deregulierung diskutiert werden.

Im zweiten Kapitel werden zunächst die bestehenden Regulierungen auf dem deutschen Apothekenmarkt vorgestellt. Der anschließende dritte Abschnitt wendet dann ökonomische Theorien auf den betrachteten Fall an, um in einem vierten Kapitel eine Bewertung vorzunehmen und schließlich die Wirkungen möglicher Deregulierungsmaßnahmen einschätzen zu können.

\section{Die Regulierung des Apothekenwesens}

Derzeit existieren in Deutschland 21.602 Apotheken, wovon 2.851 als Filialapotheken geführt werden. Mit einem Verhältnis von 3.800 Einwohnern je Apotheke ist die Apothekendichte in Deutschland relativ hoch. (ABDA, 2009)

Die Regulierung auf dem Apothekenmarkt soll eine ausreichende Zahl der Abgabestellen, ordnungsgemäße Abgabe der Arzneimittel, hochqualitative Beratung, Zugang zu Arzneimitteln zu jeder Tages- und Nachtzeit, ein angemessenes Arzneimittelpreisniveau, Vermeidung von missbräuchlichem und unsachgemäßem Konsum von Arzneimitteln sowie die Verhinderung einer marktbeherrschenden Stellung von Anbietern bzw. deren Ausnutzung sicherstellen. Zusätzlich werden als Begründung auch die Mittelstandsförderung und der Erhalt des Apothekerberufes als freier Beruf genannt (Schöffski, 1995, S. 25ff). ${ }^{2}$ Letztere Ziele entziehen sich selbstverständlich einer ökonomischen Fundierung, da sie ein Partikularinteresse aufzeigen und eher einer generellen, nur historisch erklärbaren, gesellschaftspolitischen Wertvorstellung folgen.
Die Regulierungsmaßnahmen des Apothekerwesens sind dabei im Wesentlichen im Gesetz über das Apothekerwesen (Apothekengesetz - ApoG) in Verbindung mit der Verordnung über den Betrieb von Apotheken (Apothekenbetriebsordnung ApBetrO) und der Bundes-Apothekerordnung (BApO) normiert.

Der Betrieb einer Apotheke ist an eine Erlaubnis geknüpft. Die Erlaubnis wird erteilt, wenn der Antragsteller eine Approbation (Zulassungsbeschränkung laut Approbationsordnung) vorweisen kann. Nicht-Apothekern ist die Beteiligung an einer Apotheke verwehrt, auch die erlaubten Rechtsformen sind vorgegeben (Fremdbesitzverbot). Beschränkt ist ebenfalls die Zahl der Apotheken, die ein Apotheker besitzen darf (max. vier Niederlassungen je Apotheker; Mehrbesitzbeschränkung). Hiermit eng verknüpft ist auch die Pflicht und Verantwortlichkeit zur persönlichen Leitung der Apotheke.

Neben diesen Maßnahmen zur Beschränkung des Wettbewerbs existiert eine Reihe von Einzelregelungen für den Geschäftsbetrieb und zum Sortiment einer Apotheke: Dies umfasst Regelungen bzgl. der Einhaltung von Mindestanforderungen zu Größe und Einrichtung der Apothekenräume, Anforderungen an das Personal, ein Verbot von Kooperationsverträgen, die dauernde Dienstbereitschaft, Regelungen zum In-den-Verkehr-bringen von Arzneimitteln und ,apothekenüblichen" Waren und eine Informations- und Beratungspflicht aus Gründen der Arzneimittelsicherheit. Neben diesen Regulierungen des Geschäftsbetriebs existieren zudem weitere Bestimmungen zum Sortiment einer Apotheke. Dies umfasst die Apotheken- und Verschreibungspflicht, die Beschränkung des Sortiments auf „apothekenübliche“ Waren und Regelungen zur Vorratshaltung.

Hinzu kommen noch die gesetzlichen Restriktionen des Heilmittelwerbegesetzes, die jeder Akteur des Gesundheitswesens zu befolgen hat. Insbesondere ein Verbot der Publikumswerbung für verschreibungspflichtige Arzneimittel ist hierbei von Relevanz.

Auch die Apothekenverkaufspreise für verschreibungspflichtige Arzneimittel unterliegen in Deutschland einer strengen Regulierung. Unter Berücksichtigung der MwSt. von derzeit 19\% ergibt sich der Apothekenverkaufspreis in der Regel wie folgt:

Apothekenverkaufspreis $=$ $(($ Herstellerabgabepreis + Großhandelshöchstzuschlag $) \times 1,03+$ $€ 8,10) \times(1+0,19)$

Im europäischen Kontext existieren verschiedenste nationale Normen, deren Vereinheitlichung durch das EuGH-Urteil nicht verlangt wurde. Nicht ein generelles, europaweites Verbot von Fremd- und Mehrbesitz war Gegenstand des Urteils, sondern vielmehr die Bestätigung der Zulässigkeit individueller, nationaler Regelungen. 


\section{Wirtschaftstheoretische Analyse des Arzneimittelmarktes}

\subsection{Wo ist mehr Wettbewerb, im Polypol oder Oligopol?}

Der deutsche Apothekenmarkt kann als ein besonderer Fall eines Polypols klassifiziert werden: eine Vielzahl von unabhängigen Anbietern trifft auf eine Vielzahl von Nachfragern. Der Marktanteil jedes einzelnen Anbieters ist so klein, dass Veränderungen von Preis (in zulässigen Nischen) oder der Menge (bzw. der Qualität der erbrachten Dienstleistung) von einzelnen Apotheken keinen merklichen Einfluss auf die Marktsituation der übrigen Konkurrenten hat. Polypolistische Konkurrenz- oder Wettbewerbsmärkte führen allerdings nur dann zu einer paretooptimalen Allokation (und damit zur größtmöglichen gesellschaftlichen Wohlfahrt), wenn vollkommene Transparenz und keine die Entscheidungsfreiheit des Einzelnen beschränkenden Regulierungen bestehen (Wied-Nebbeling, 1997, S. 9). Der deutsche Apothekenmarkt ist aber durch eine Vielzahl gesetzlicher Eingriffe gekennzeichnet. Insbesondere der freie und unbeschränkte Marktzutritt ist in Deutschland nicht gegeben.

Durch die vereinzelt in Deutschland angedachten Deregulierungsmaßnahmen wäre hingegen ein Trend zu einer - je nach Region - mehr oder minder oligopolistischen Marktstruktur zu erwarten. Gerade Anbieter, die bereits in oligopolistisch geprägten Märkten tätig sind (z.B Pharmagroßhändler und Drogerieketten), könnten ihre Erfahrungen im Sinne einer Marktintegration nutzen, um in das Apothekengeschäft vorzustoßen. ${ }^{3}$

\subsection{Anwendung von Oligopolmodellen auf den Apothekenmarkt}

In der wirtschaftswissenschaftlichen Theorie existieren verschiedene Ansätze, um die Wirkweisen und Konsequenzen des oligopolistischen Wettbewerbs zu beschreiben. Diese Oligopolmodelle und deren Wirkungen unterscheiden sich vornehmlich darin, welche Größe sie als den langfristig strategisch ausschlaggebenden Aktionsparameter annehmen (Preis, Angebotsmenge, Produktdifferenzierung etc.) und ob von Homogenität oder Heterogenität des Angebotes ausgegangen wird. Oligopol-Märkte sind zu vielem fähig: Von friedlicher und gemeinsamer Koexistenz der Marktteilnehmer bis hin zum erbitterten Preiskrieg (WiedNebbeling, 1997, S. 140f.). Die für den vorliegenden Fall relevanten Modelle sollen kurz beschrieben werden:

\section{Das Bertrand-Modell}

Anders als im sogenannten Cournot-Modell (Cournot, 1838) (Mengenwettbewerb: Anbieter entschieden simultan über die Angebotsmengen; hieraus ergibt sich ein Preis, welcher Angebot und Nachfrage ausgleicht) wird im Bertrand-Modell (Bertrand, 1883) angenommen, dass die Anbieter den Preis setzen und zu diesen Preisen beliebige Mengen liefern können - was in der Regel auf dem Apothe- kenmarkt der Fall sein dürfte. Voraussetzungen sind eine hohe Markttransparenz und die Abwesenheit von Kundentreue („Präferenzen“) mit der Folge, dass alle Nachfrager nur beim preisgünstigsten Anbieter kaufen. Entscheidend ist dabei auch, dass der Nachfrager den Preis vollständig selber zu tragen hat (Pfähler/ Wiese, 2005, S. 126f.; Varian, 2004, S. 500f.). Wenn die Produkte perfekte Substitute sind (homogenes Oligopol), ist das Unterbieten eines bestehenden Preises für den einzelnen Oligopolisten lohnend und der Marktpreis in der Folge entsprechend niedrig. Ergebnis ist eine Preissetzung auf Höhe der Grenzkosten, so dass die Gewinne der Oligopolanbieter auf (konkurrenz-) marktübliche Werte sinken. An diesem Punkt hat keiner der Anbieter Anreize, den gewählten Preis zu verändern, da eine Erhöhung mit dem Verlust der evtl. gesamten Nachfrage einherginge und eine Preissenkung eigene Verluste je verkaufter Einheit zur Folge hätte. Dieses für die Kunden optimale Ergebnis (pareto-optimales Gleichgewicht) kann schon bei nur zwei Marktteilnehmern erreicht werden. Das Bertrand-Oligopolmodell verdeutlicht somit, dass nicht allein die Zahl der Marktteilnehmer über Ergebnis und Stärke des Wettbewerbs entscheiden.

Für den Apothekenmarkt kann man - bei entsprechend weiter Deregulierung auch der Preissetzung - durchaus davon ausgehen, dass sich die Oligopolisten zunächst in Richtung eines Bertrand 'schen Preiswettbewerbs bewegen würden. Insbesondere gilt das für den Bereich der nichtverschreibungspflichtigen Präparate, in dem die Kundensouveränität bereits heute relativ stark ausgeprägt ist: Der Kunde hat zwar freie Medikamentenwahl muss aber die Kosten in vollem Umfang selbst tragen. Im Sinne eines Erfahrungsguts wird der Kunde hier mit der Zeit mehr und mehr selbst zum informierten (und damit selbstbestimmten) Entscheider und muss weniger auf die Serviceleistung der Beratung durch den Apotheker zurückgreifen. Besonders ein relativ homogener Markt, welcher in der ersten Zeit nach Deregulierung zu erwarten wäre, ist für Preiskämpfe (insbesondere im OTC-Bereich) empfänglich.

\section{Das Sweezy-Modell}

Aus wohlfahrtstheoretischer Perspektive würde in diesem Fall kein Anlass mehr bestehen, den Markt weiter zu regulieren. Lediglich kartellrechtliche Aufsicht wäre vonnöten. Eine Gefahr bestünde lediglich darin, dass es unter den Oligopolisten zu gar keinem Wettbewerb kommt, da die Marktteilnehmer invariat gegenüber bestimmten Änderungen sein können und eine eventuel zwischenzeitlich erreichte Gleichgewichtsposition nicht verlassen. Genau dieses Phänomen versucht das Oligopolmodell nach Sweezy mit der geknickten Preis-Absatz-Kurve zu beschreiben (Sweezy, 1939; vgl. zusätzlich Schumann/ Meyer/ Ströbele, 2007, S. 361ff.). In dem Ansatz rechnet der Anbieter damit, dass seine Preisanhebungen zu starken Absatzeinbußen führen, da Konkurrenten die Preisanhebung nicht mitmachen. Eine Preissenkung führt demgegenüber nur zu einem geringen Absatzgewinn, da die Konkurrenz in diesem Falle mitziehen würde. Letztendlich wird ein Oligopolmarkt beschrieben, auf dem der Preis als Wettbe- 


\section{THEMA}

werbsoption der Teilnehmer entfällt, da dieser quasi starr bleibt und somit nur Größen wie Werbung und Serviceleistungen entscheiden.

Geht man zunächst davon aus, dass lediglich Fremd- und Mehrbesitzverbot fallen, ohne die bestehende Preisregulierung und Vollversicherung anzutasten, so könnte dieses Modell die sich ergebende Situation auf dem Apothekenmarkt beschreiben. Ein intensiver Qualitätswettbewerb bei weiterhin regulierten Preisen wäre die Folge. Apotheken würden beispielsweise versuchen, sich über die Servicequalität auszudifferenzieren. Hierbei muss jedoch angemerkt werden, dass in der ursprünglichen Erklärung des Modells der fixe Preis eine Folge des Oligopols ist und nicht aus rechtlichen Restriktionen des Gesetzgebers erwächst und damit unabhängig vom Oligopol besteht.

Die Folge wäre dennoch ein mäßig intensivierter Wettbewerb. Es ist offensichtlich, dass dieser Fall nicht zu einer pareto-optimalen (wenn im Vergleich zum Status Quo aber pareto-verbessernden) Marktsituation führen wird. Umso deutlicher kann an dieser Theorie erkannt werden, wie wichtig die gleichzeitige Aufhebung von Preisregulierungen, Vollversicherungsmentalität und Beseitigung aller nicht für die Sicherheit erforderlichen Regulierungen ist. Auch Wettbewerbsanreize, welche z.B. den Abschluss von Einzelverträgen zwischen Apotheken(-ketten) und Krankenversicherern genehmigen, können in der Lage sein, ein Sweezy-Oligopol zu vermeiden und so eine hohe Wettbewerbsintensität zu fördern.

\section{Das Hotelling-Modell}

Langfristig steht zu erwarten, dass der zunächst relativ homogene Markt zumindest ein Stück weit heterogener werden wird. Das so genannte „Straßenmodell“ nach Hotelling (Hotelling, 1929) stellt dabei einen geeigneten Ansatz zur Analyse und Prognose der langfristigen Folgen einer Deregulierung des Apothekenmarktes dar. Dieses Oligopolmodell stellt eine Modifikation des Bertrand-Modells dar (Erweiterung um Differenzierung, d.h. heterogene Güter für die ebenfalls heterogenen Präferenzen der Kunden).

Insbesondere auf oligopolistischen Märkten spielen spezifische Nachfragerpräferenzen bezüglich einzelner Marken, Angebots-, Vertriebs- und Servicekonzepte eine wichtige Rolle. ${ }^{4}$ Hierbei lässt sich die Hotelling-Straße als eine Art „Geschmacksstrecke“ vorstellen, auf der Produkte angeboten werden. Die fiktive Entfernung eines Kunden zum angebotenen Produkt gibt dabei an, wie weit dieses von der gewünschten Ausprägung entfernt ist. Kunden, deren Präferenzen nicht exakt getroffen werden, entstehen „Transportkosten“ im Sinne eines Nutzenverlustes. Der Konsument wird bei gleichen Marktpreisen denjenigen Anbieter wählen, der ihm am meisten gefällt, d.h. zu dessen „Laden“ er die kürzeste präferenzmäßige Distanz zurückzulegen hat. Bei differierenden Preisen spielen dagegen sowohl Preisunterschied als auch Transportkosten eine Rolle (Wied-Nebbeling, 1997, S. 206).
Das Modell passt gut für die Erklärung der langfristig zu erwartenden Marktstruktur und -dynamik auf dem Apothekenmarkt nach einer Deregulierung. Sowohl traditionelle Apotheken als auch „Marken“-Apotheken, die von Kapitalgesellschaften betrieben werden, wie auch Apotheken mit neuen Vertriebs- und Servicekonzepten werden sich ausdifferenzieren, sodass der Differenzierungsgrad ansteigen wird. Die Produktheterogenität im Apothekenbereich besteht dabei in erster Linie in den Bereichen der Beratungsqualität und -freundlichkeit, der Personalausstattung, der zeitlichen und örtlichen Erreichbarkeit, speziell eingerichteten Räumlichkeiten für Beratungen oder kleinere Untersuchungen (wie Blutdruckmessen) oder auch in der Option eines Express- bzw. Frei-Haus-Lieferservices. Langfristig werden sich verschiedene Modell herausbilden und verschiedene Formen der Arzneimitteldistribution können in Koexistenz bestehen. Verschiedene Anbieter mit verschiedenen Konzepten (Qualitätsführerschaft, Preisführerschaft, geografische Nische, Anbieten von eigenen Handelsmarken z.B. im OTC-Bereich etc.) erlauben eine wohlfahrtssteigernde Produktvielfalt bei gleichzeitig hohem Wettbewerbsdruck in vielen der - in sich wiederum homogenen - Marktnischen. Eine den tatsächlichen Präferenzen entsprechende Ausgestaltung bzw. Verteilung der Produktdifferenzierung würde wohlfahrtssteigernd sein, da „Transportkosten“ der Menschen auf diese Weise minimiert werden.

Der sich tatsächlich ergebende Differenzierungsgrad ist abhängig von einer Vielzahl von Faktoren, z.B. der Präferenzen der Kunden, der Preiselastizität der Nachfrage oder der Ausgestaltung der „Transportkostenfunktion“. Es werden im Übrigen so lange Anbieter mit weiteren Formen des Angebotes in den Markt eintreten, bis die zusätzlichen Produktionsfixkosten nicht mehr durch eine zusätzlich generierte Zahlungsbereitschaft gedeckt werden (Woeckener, 2006, S. 149ff.). Der Markt führt so zu einem Gleichgewicht.

\subsection{Kurz- und langfristige Wirkungen der Deregulierung}

Zusammenfassend ist zu erwarten, dass die umfassenden Deregulierungsmaßnahmen relativ kurzfristig Tendenzen zu einem homogenen Oligopol und Bertrand'schen Preiswettbewerbsmechanismen auslösen werden (v.a. im Bereich der nicht-verschreibungspflichtigen Medikamente). Die angebotenen Güter sind aus Sicht der Kunden nahezu perfekte Substitute, es bestehen demzufolge keinerlei Präferenzen für Güter unterschiedlicher Anbieter, da jeder dasselbe und niemand „eigene“ oder exklusive Produkte anbietet. Sinkende Preise und eine gesteigerte gesellschaftliche Wohlfahrt sind die Folge. Je größer der Deregulierungsumfang (insbesondere der Preisregulierung), umso mehr dieser Wohlfahrtssteigerungen sind zu erwarten.

Bleibt jedoch die weitgehend starre Preisregulierung im verschreibungspflichtigen Segment bestehen, wird sich der Wettbewerb in diesem Bereich des Apothekenmarktes (nur) in Richtung Qualitätswettbewerb orientieren. Dieser umfasst die Beratungsqualität, zusätzliche Serviceleistun- 
gen oder Gesundheits-Checks. Die Abschaffung der Preisregulierung inklusive einer Minderung der Vollversicherung auch im verschreibungspflichtigen Segment würde jedoch die Wettbewerbsdynamik zusätzlich verstärken.

Nach gewisser Zeit wird sich das Wettbewerbsumfeld so aufgrund unterschiedlicher strategischer Stoßrichtungen heterogener entwickeln. Zwar bleiben die eigentlichen Produkte homogen, jedoch entwickeln sich nach und nach bestimmte Differenzierungen zwischen den unterschiedlichen Anbietern hinsichtlich dem Image, beim Preisniveau (zumindest in dem Bereich, in dem hierfür gesetzlicher Spielraum eingeräumt wird), der Service- und Beratungsqualität, der geographischen Lage und dem Einsatz eines absatzpolitischen Instrumentariums. Unter Berücksichtigung der von Hotelling in seinem Straßenmodell vorhergesagten Effekte, kommt es hierdurch zu einem langfristigen Gleichgewicht, welches ein höheres Wohlfahrtsniveau für die Apothekenkunden ermöglicht als die derzeitige Situation. Risiken einer oligopolistischen „Reaktionsverbundenheit" und daraus evtl. entstehende marktbeherrschende Stellungen müssen durch Anwendung des Wettbewerbsrechts eingedämmt werden. Je weitgehender jedoch die Deregulierung ist, desto unwahrscheinlicher ist der Fall abgestimmten Verhaltens der Anbieter.

\section{Bewertung der derzeitigen Regulierungsmaßnahmen in Deutschland}

\subsection{Maßnahmen zur Begrenzung des Wettbewerbs}

Nach dieser recht allgemeinen wirtschaftstheoretischen Analyse sollen nun die einzelnen konkreten Bestandteile der Regulierung des deutschen Apothekenmarktes einer Bewertung unterzogen werden. Hierfür ist es zunächst notwendig, die Leitung und das Eigentum einer Apotheke separat zu betrachten.

Die Leitung durch einen Apotheker lässt sich mit Blick auf die potenzielle Gefährlichkeit von Arzneimitteln bei unsachgemäßer Anwendung medizinisch und ökonomisch rechtfertigen. Der Schutz des (in der Regel uninformierten) Verbrauchers legitimiert die Leitung durch einen Experten mit höherem Informationsstand. Hierdurch wird einer fehlerhaften Verwendung von Arzneimitteln vorgebeugt, dessen Folgekosten darüber hinaus von der Gesellschaft zu tragen wären (beispielsweise in Form noch höherer Versorgungskosten oder auch durch schwindendes Vertrauen in eine sichere und geordnete Arzneimittelversorgung). Deshalb ist durchaus begründbar, dass in einer Apotheke ein Apotheker die Abgabe von Arzneimitteln überwacht. Auch verlangt das Ziel der Gewährleistung einer hochqualitativen Beratung, dass die Apotheke mit entsprechendem Fachpersonal ausgestattet ist.

Der zweite Teil der Vorschrift, nämlich dass eine Apotheke im Besitz eines ausgebildeten Apothekers zu sein hat, lässt sich hingegen weder mit den angeführten Zielen noch mit Hilfe der ökonomischen Theorie oder Empirie begründen. Auch sind auf absehbare Zeit keine Konzentra- tionstendenzen zu erwarten, die zu marktbeherrschenden Stellungen führen würden. Die Kostenfunktion bei Apotheken ist ähnlich der von Einzelhandelsunternehmen. Es liegt auch ausdrücklich nicht der Fall des natürlichen Monopols vor, dem stets die Gefahr von Kartellierungen innewohnt. ${ }^{5}$ Vielmehr ist nach Deregulierung die Entwicklung einer Marktstruktur zu erwarten, in der sich eine große Zahl von Anbietern in einem intensiven Wettbewerb gegenübersteht. Dies würde jedoch weder die traditionell durch einen Apotheker betriebene Apotheke komplett verdrängen, noch den Wettbewerb unter Apotheken vermindern. Wie das Beispiel des Optikermarktes zeigt, bleiben selbstständige Einzelhändler nicht auf der Strecke: Obwohl es bundesweit tätige Ketten gibt, zeichnen sich Einzelgeschäfte häufig durch eine besondere Kundenbeziehung aus, die vor allem auf Vertrauen basiert. Das Geschäft der inhabergeführten Apotheken wird sich also grundlegend ändern, zwangsläufig ist ihr Ausscheiden jedoch keinesfalls. (ZEIT, 2006)

Das Fremdbesitzverbot wird oftmals auch damit begründet, dass ein Eigentümer-Apotheker für fehlerhafte Abgaben haftet und aus Eigeninteresse dafür sorgt, dass keine Haftungsfälle entstehen (Glaeske/ Klauber/ Lankers/ Selke, 2003, S. 123ff). Hierbei wird jedoch übersehen, dass ein angestellter Apotheker ebenfalls haftet. Zudem kann auch eine Haftung für den neuen finanzstarken Eigentümer der Apotheke entstehen. Ebenso ist der Hinweis auf den hohen Wert der Marke bzw. des Markennamens (in Verbindung mit einer Markenqualität) für Apothekengroßbetriebe an dieser Stelle sinnvoll: Sollte es in einer Filiale einmal Probleme geben, so strahlt diese negative Wirkung sogleich auf andere Mitglieder des Verbundes ab. Allein zum Schutz der Reputation werden große Kapitalgesellschaften alles tun, um Risiken zu vermeiden und Haftungsfälle gar nicht erst auftreten zu lassen.

Auch ist nicht zu erwarten, dass bei Aufhebung des Fremd- und Mehrbesitzverbotes die Zahl der Arzneimittelabgabestellen stärker sinkt, als dies aufgrund der ohnehin zu erwartenden Marktbereinigung sinnvoll wäre. Derzeit wird die Arzneimitteldistribution zu überhöhten volkswirtschaftlichen Kosten betrieben: Ökonomisch eigentlich zu kleine Betriebe können aktuell bestehen, da sie durch effizientere Betriebe nicht genügend unter Druck gesetzt werden können. Gegebenenfalls erfolgt dieser Anpassungsprozess durch eine Deregulierung nur schneller. Viele derzeitige Apotheken sind aus betriebswirtschaftlichen Gründen nicht mehr sinnvoll zu betreiben und werden deshalb von den Betreibern mittelfristig aufgegeben werden (Monopolkommission, 2006, S. 416 Ziffern 1151ff.). Kapitalgesellschaften, die eine Vielzahl von Apotheken betreiben, können hingegen Skaleneffekte in allen betrieblichen Funktionen realisieren und sind daher bei gegebenen Preisen eher in der Lage, Überschüsse zu erzielen und Apotheken auch in nicht bevorzugten Lagen zu betreiben.

Die Wirkungen von Regulierungs- und Deregulierungsmaßnahmen sollten jedoch nicht isoliert betrachtet werden: So führt ein Aufheben der Fremd- und Mehrbesitzregelung bei gleichzeitigem Fortbestand der Preisregu- 


\section{THEMA}

lierung zu anderen Effekten als bei einem gleichzeitigen Aufheben der Arzneimittelpreisverordnung (AMPreisV) in ihrer jetzigen Form. Wie im vorherigen Kapitel beschrieben, wird im ersteren Fall der Anpassungsprozeß langsamer erfolgen und der Wettbewerb vor allem den Charakter eines Qualitätswettbewerbs haben. Im zweiten Fall treten sowohl ein Preis- als auch ein Qualitätswettbewerb ein, wobei sich Anbieter durch regionale Preisdifferenzierung auch regionalen Kostenunterschieden anpassen können. Im Ergebnis wird sich hierdurch eine gleichmäßigere regionale Verteilung der Apotheken ergeben, da Apotheker in bislang weniger gut versorgten und damit konkurrenzarmen Gebieten die Freiheit hätten, die Preise an die relative Knappheit von Apothekenkapazitäten anzupassen.

\subsection{Regulierung des Geschäftsbetriebs}

Das Gebot der Missbrauchsverhinderung beim Konsum von Arzneimitteln ist geeignet die Anforderungen an die Führung der Apotheke sowie die Regelungen zum „In-denVerkehr-bringen" von Arzneimitteln und apothekenüblichen Waren, die Apothekenpflicht und die Verschreibungspflicht zu rechtfertigen. Für die Forderung nach räumlicher Abgeschlossenheit der Apotheke spricht, dass so die ordnungsgemäße Abgabe von Arzneimitteln sichergestellt werden kann. Auch die Beschränkung auf den Verkauf „apothekenüblicher" Waren ist eine konsistente Auflage im Rahmen der derzeitigen Regulierung.

Viele der anderen genannten Regelungen sind dagegen heutzutage nicht mehr erforderlich, da die in ihnen formulierten Anforderungen ohnehin von den Anbietern aufgrund des Wettbewerbs erfüllt würden. Dazu gehören die Dienstbereitschaft, die Qualifikation des Personals, die Ausstattung mit Apparaturen und die Vorratshaltung. Durch gemeinsame Nutzung eines telefonischen Bereitschaftsund Belieferungsdienstes sowie von Geräten wäre dies besser zu organisieren. Größere Einheiten, beispielsweise in Form von Kapitalgesellschaften, werden diese Möglichkeiten der Nutzung von Wirtschaftlichkeitspotentialen eher wahrnehmen als Einzelapotheker. Verschwendung volkswirtschaftlicher Ressourcen würde durch Deregulierung vermieden. ${ }^{6}$

Angesichts der Tatsache, dass Apotheken heute zum größten Teil industriell gefertigte Arzneimittel abgeben, können auch die Anforderungen an die Größe der Apothekenräume aktualisiert werden: Eine Apotheke, die den Anspruch hat, eine umfassende Versorgung der Patienten mit Arzneimitteln sicherzustellen, kann eine bestimmte Größe sowieso nicht unterschreiten.

\subsection{Preisregulierung}

Auf dem deutschen Arzneimittelmarkt wird der Preismechanismus zur Koordination von Angebot und Nachfrage derzeit nur in Ansätzen genutzt. Stattdessen sind die Preise für verschreibungspflichtige Medikamente durch die Arzneimittelpreisverordnung weitgehend festgelegt.
Wie ausgeführt wurde, verlangen die gesundheitspolitischen Ziele, dass eine Marktordnung des Apothekenwesens erstens eine ausreichende Anzahl von Arzneimittelabgabestellen und zweitens den Schutz des Verbrauchers vor überhöhten Preisen sicherstellt.

Zunächst ist festzustellen, dass beide Ziele durch die gegenwärtige Regulierung erreicht werden. Schließlich sind die Preise nach oben begrenzt und auch die Apothekendichte mit 3.800 Einwohnern pro Apotheke (ABDA, 2009) (über-)erfüllt die Anforderungen an eine ausreichende Anzahl von Abgabestellen.

Auf der anderen Seite ist allerdings auch jede Form der Preisregulierung sorgsam zu begründen und stets zu prüfen, ob nicht weniger regulatorische Eingriffe ebenfalls geeignet wären, das angestrebte Ziel zu erreichen. Bezüglich der Preisregulierung ist dabei zu bemerken, dass in der Regel die Allokation der Ressourcen durch eine Preisregulierung nicht verbessert, sondern verschlechtert wird und die häufig postulierten distributiven Ziele ebenfalls nicht erreicht werden: Sind die durch die Preisregulierung festgelegten Preise zu gering, kommt es zu Versorgungsengpässen, Warteschlangen und offener bzw. versteckter Rationierung. Sind sie zu hoch, dann sind überhöhte Gewinne, Verschwendung und Überversorgung die Folge. Eine Freigabe der Preise würde hingegen den Wettbewerb fördern, müsste aber in beide Richtungen erfolgen, wenn die Preisregulierung nicht nur als Rationierungs- und Kostendämpfungsinstrument gesehen werden soll.

Im nun folgenden Abschnitt sollen einige bereits tangierte Überlegungen zu Verbund- und Skaleneffekten im Rahmen der betriebswirtschaftlichen Transaktions- und Produktionskostentheorie eingeordnet und auf den Apothekenmarkt angewandt werden.

\subsection{Effizienzüberlegungen zur Betriebsgröße - Produktions- und Transaktionskostenansatz}

Die Marktmacht größerer Unternehmen auf den InputMärkten ist zur Realisierung positiver Effekte ein wichtiger Faktor. Mit zunehmender Nachfragemacht können die Einkaufspreise bzw. -konditionen auf den Faktormärkten verbessert werden. Der maßgebliche Faktor bei den Verhandlungen ist der Umsatz, den eine Apotheke dem Lieferanten anbieten kann (Kierndorfer, 2007, S. 31). Größere wirtschaftliche Einheiten können z.B. ihre Verhandlungsstärke gegenüber den Pharmagroßhändlern und -herstellern deutlich erhöhen. Für einzelne Apotheken ist es aus logistischen Gründen hingegen häufig schwieriger, Marktmacht auszuüben (Blohorn, 2006, S. 11). Auch werden den Herstellern durch Konzentrationen auf der Handelsebene Wahlmöglichkeiten genommen und aufgrund der großen Abnahmemenge der entstehenden Großapotheke können diese eine Verringerung des Distributionsangebotes erfolgreich androhen (Niestraht, 1983, S. 147f.).

Hinzu kommen Spezialisierungsvorteile bzw. Vorteile der Arbeitsteilung: Je größer die produzierte Ausbringungsmenge eines Unternehmens ist, desto effizienter lassen 
sich spezialisierte Leistungsfaktoren durch eine Aufspaltung des Arbeitsprozesses in einzelne Teilaktivitäten aufschlüsseln. Die Folge ist eine Produktivitätssteigerung aufgrund der Herausbildung von Spezialisten für bestimmte Aufgaben (Lindstädt/ Hauser, 2004, S. 12).

In einem kleinen Apothekenbetrieb wird dies kaum effizient zu leisten sein. Erst in einem Apothekenbetrieb entsprechender Größe wird es sinnvoll, den Teil des Leistungserstellungsprozesses bei dem nur wenig direkter Kontakt zum Kunden besteht, weiter aufzuspalten und die entsprechenden Teilprozesse mit Spezialisten zu besetzen.

Auch die technischen Vorteile, Fixkostendegressionen und die besseren Möglichkeiten zur Risikostreuung größerer Einheiten sind zu beachten. Bei größeren Produktionsmengen können Produktionstechnologien oder -methoden eingesetzt werden, die einen verbesserten Ressourceneinsatz ermöglichen (Bühler/ Jäger, 2002, S. 19f.). Konstante Fixkosten unteilbarer Ressourcen können so auf eine größere Stückzahl von Erzeugnissen verteilt werden (Lindstädt/ Hauser, 2004, S. 12). Degressionseffekte entstehen dabei hauptsächlich aufgrund der Zentralisierung von Rechnungswesen und Controlling, Personalmanagement, Marketing oder auch durch einen gemeinsam betriebenen 24-h-Service bzw. -Lieferbereitschaft.

Zudem sind transaktionskostentheoretische Betriebsgrößenvorteile von Bedeutung. Eine Kapitalgesellschaft, die eine Vielzahl von Apotheken betreibt, kann betriebliche Funktionen in einer Zentrale bündeln und steuern. Ein Apothekensystem wird umso effizienter sein, je mehr Kontakte es zu bestehenden und potentiellen Transaktionspartnern einsparen kann und je größer die Skalenerträge durch Bündelung von Transaktionen sind. Damit sinken nicht nur auf der Abnehmerseite die Transaktionskosten, sondern auch auf der Lieferantenseite (z.B. da auch diese lediglich einen zentralen Ansprechpartner haben).

Mikroökonomisch betrachtet, handelt es sich bei all diesen Vorteilen um so genannte positive Skalen- oder Verbundeffekte. Einerseits werden horizontale Integrationen - also Zusammenschlüsse von Apotheken - diese Effekte in der Arzneimitteldistribution auslösen. Andererseits aber auch vertikale Integrationen, also beispielsweise ein Zusammenschluss von Großhandelsunternehmen mit Apothekenbetriebsunternehmen. Verbundvorteile, beispielsweise von „Shop-im-Shop“-Konzepten, entstehen durch die gemeinsame Nutzung einer hohen Kundenfrequenz, der Ladenflächen oder des Personals.

Gegen eine Aufhebung der Vorschriften zur Beschränkung der Apothekenbetriebsgröße könnte nun eingewandt werden, dass sich entsprechende Größenvorteile auch durch inhabergeführte Kleinapotheken mittels einer Einbindung in Kooperationssysteme erzielen ließen. Dies stimmt und wird auch als Reaktion auf von Kapitalgesellschaften betriebene Apotheken ggf. so kommen. Unter den aktuell gegebenen gesetzlichen Vorschriften wäre dabei allerdings lediglich ein System mit eher geringer Bindungsintensität die Folge. Eine verstärkt bindungsintensive hierarchische Koordinationsform (bestehend aus einer Systemzentrale und diversen Mitgliedsapotheken) besitzt jedoch entscheidende Vorteile beim Ausschöpfen der Skalen- und Verbundeffekte: In der Zentrale vorhandene Managementkapazitäten und Größenvorteile können umfassender genutzt und Kostensenkungspotentiale noch besser ausgeschöpft werden. Eigeninitiativen der Mitglieder können in einem solchen System besser koordiniert bzw. unterbunden werden: Durchsetzungs- und Kontrollaufwand sind deutlich geringer.

Eine Beschränkung der Apothekenbetriebsgröße kann vor diesem Hintergrund ökonomisch nicht gerechtfertigt werden. Die Überlegenheit eines Apothekengroßbetriebs gegenüber relativ bindungsschwachen Kooperationen kann angenommen werden. Neben der optimalen Größe eines Apothekensystems sollte auch die optimale Koordinationsform desselbigen durch Wettbewerb in einem Versuchs- und Entdeckungsverfahren bestimmt werden. Der Ausschluss bestimmter Koordinationsformen (wie bindungsintensiver Filialsysteme) vom marktinhärenten Findungsprozess, ist unter ökonomischen Gesichtspunkten nicht zu legitimieren. Insgesamt ist offensichtlich, dass sich diese Potentiale erst ab einer bestimmten Betriebsgröße einstellen werden. Die Zahl von vier Niederlassungen je Apotheker ist in der Regel zu klein, um die genannte Effekte realisieren können.

\subsection{Notwendigkeit zur Deregulierung}

Die herausgearbeiteten Deregulierungsnotwendigkeiten sind in der nachfolgenden Tabelle zusammengefasst.

- Das Mehrbesitzverbot von Apotheken bzw. seine Beschränkung auf vier Apotheken ist ökonomisch nicht zu rechtfertigen, verhindert Kostenersparnisse aufgrund von 'economies of scale' und behindert die Entwicklung innovativer Ansätze der Vertriebs- und Beratungstätigkeit von Apotheken. Dazu vermindert es die Wettbewerbsintensität.

- Eine Beseitigung des Fremdbesitzverbotes würde dazu führen, dass die Apotheken im Durchschnitt über ein höheres Haftungskapital verfügen und auch im Management von Apotheken moderne betriebswirtschaftliche Methoden zur Anwendung kommen. Die Kapitalallokation würde im Bereich der Arzneimitteldistribution verbessert.

- Die Abgeschlossenheit von Apotheken erscheint dann nicht zwingend erforderlich, wenn entsprechende Konzepte entwickelt werden könnten, die die Versorgung, Beratung und Missbrauchsverhinderung dennoch sicherstellen. Derzeit liegen jedoch derartige Konzepte nicht vor.

- Eine Rückführung der Preisregulierung würde das Erreichen der gesundheitspolitischen Ziele nicht unmittelbar gefährden und den Wettbewerb stärken. Angesichts der Dominanz des Kostendämpfungszieles in der Gesundheitspolitik ist hier jedoch keine Bewegung zu erwarten. 


\section{Folgen einer Deregulierung des Apothekenmarktes}

Die Auswirkungen einzelner Deregulierungsschritte auf das Apothekenwesen sollen nun analysiert werden. Dabei betrachten wir zwei Szenarien: Eine Deregulierung der bestehenden Mehr- und Fremdbesitzregelungen ohne Beseitigung der Vollversicherung und Festpreise gegenüber einer Deregulierung inklusive dieser beiden Reformschritte.

\subsection{Deregulierung der Arzneimitteldistribution bei be- stehender Vollversicherung und Festpreisen}

\subsubsection{Wirkung einer Abschaffung der Vorschriften zum Mehrbesitz von Apotheken}

\section{Alleinige Änderung der Mehrbesitzregelung:}

Eine Abschaffung der Mehrbesitzregelung gäbe Apothekern mit einer Hauptapotheke und drei Filialen die Möglichkeit zur Ausweitung des Geschäfts. Es ist jedoch nicht zu erwarten, dass viele Apotheken diese Möglichkeit nutzen würden. Ende des Jahres 2008 gab es 21.602 Apotheken (wobei die Zahl über die Jahre recht konstant ist), hiervon allerdings nur 2.851 Filialbetriebe (ABDA, 2009). Meldungen, dass über Franchise-Modelle in kurzer Zeit hunderte von neuen Lizenzen vergeben werden, scheinen so lange übertrieben, wie das Fremdbesitzverbot Aufrecht erhalten bleibt.

Die ökonomische Theorie liefert den Grund für diesen empirischen Befund: Die Grenzkosten steigen offensicht- lich mit jeder weiteren Apothekenfiliale, wenn sie von einem Apotheker und nicht einer professionellen Betreibergesellschaft betrieben wird. Die zunehmenden Kosten für den Apotheker, eine weitere Apotheke zu betreiben, werden nicht durch Einsparungen von Fixkosten des gemeinsamen Betreibens einer größeren Anzahl von Apotheken überkompensiert. Dies kann mit den Schwierigkeiten eines Apothekers begründet werden, mehrere Apotheken gleichzeitig zu führen, und mit den hohen Kosten, die ein angestellter Apotheker im Vergleich zur „Selbstausbeutung“ des selbstständig tätigen Apothekers verursacht. Die ‘economies of scale`' (Kostendegression aufgrund der Größe eines Filialsystems) sind also geringer als die Kostensteigerungen des Betreibens von Filialen.

Lockerung der Anforderungen zur Abgeschlossenheit bei gleichzeitigem Fortbestand der Fremdbesitzregelungen:

Eine Lockerung der Anforderungen an die Abgeschlossenheit von Apotheken hätte zur Folge, dass es Apothekern möglich wäre, ihre Apotheken z.B. zu „Fachabteilungen“ in Kaufhäusern umzufunktionieren, wie dies in den USA der Fall ist. In Abhängigkeit der Quersubventionierung zwischen den Bereichen Nicht-Apotheken- bzw. Apothekengütern wäre es Apothekern möglich, die zusätzlichen Kosten einer Filiale, die sich insbesondere aus der Abgeschlossenheit der Geschäftsräume ergeben, zu reduzieren. Außerdem könnte die Lage in einem Kaufhaus zu einem erhöhten Absatz führen. Insgesamt werden diese Kostenvorteile aber eher gering sein und eine derartige Deregulierung erscheint in den nächsten Jahren aus

Tabelle 1: Deregulierungsnotwendigkeiten im Apothekenwesen

\begin{tabular}{|l|l|l|}
\hline \multicolumn{2}{|l|}{ Bestehende Regulierung } & Alternative Regulierung \\
\hline Anzahl der Marktteilnehmer & \multicolumn{2}{l}{} \\
\hline Fremdbesitz inkl. freier Rechtsformwahl & Nicht zulässig & Nicht notwendig \\
\hline Mehrbesitz & Begrenzt & Nicht notwendig \\
\hline Ausbildung & Vorgeschrieben & Keine Veränderung \\
\hline Geschäftsbetrieb & u. a. Abgeschlossenheit & Partielle Deregulierung möglich \\
\hline Größe und Einrichtung der Räume & Vorgeschrieben & Keine Veränderung \\
\hline Anforderungen an das Personal & Vorgeschrieben & Alternative Modelle denkbar \\
\hline Dienstbereitschaft & \multicolumn{2}{|l|}{} \\
\hline Produkte & Vorgeschrieben & Alternative Modelle denkbar \\
\hline Vorratshaltung & Vorgeschrieben & Keine Veränderung \\
\hline Apothekenpflicht & Vorgeschrieben & Keine Veränderung \\
\hline Verschreibungspflicht & \multicolumn{2}{l}{} \\
\hline Preise & Preishöchstgrenzen und & Deregulierung möglich \\
\hline Preisbildung und Preisspannen & regulierte Preisspannen & (möglichst symmetrisch) \\
\hline
\end{tabular}


Verbraucherschutzgründen weder wünschenswert noch wahrscheinlich.

\section{Einfachere Zulassung von Versandapotheken:}

Wenn der Gesetzgeber einer vereinfachten Zulassung von Versandapotheken zustimmt, ohne zugleich die Kostenerstattungsregelungen zu verändern, ist keine signifikante Änderung für die bestehende Apothekenlandschaft zu erwarten. Selbst wenn Versandapotheken einen komparativen Kostenvorteil bei der Distribution von Arzneimitteln (z.B. aufgrund einer durchschnittlich günstigeren Logistik) realisieren würden, können sie diesen Vorteil nicht an die Kunden weitergeben. Der Wettbewerbsvorteil von Versandapotheken bliebe auf die von manchen Kundengruppen bevorzugte Bestellung über das Internet und die Distribution von Arzneimitteln auf dem postalischen Weg beschränkt. Angesichts der hohen Bevölkerungs- und Apothekendichte in Deutschland ist dieser Vorteil von geringer Bedeutung. So sind die früheren Erwartungen des Bundesverbandes Deutscher Versandapotheken, dass der Marktanteil der Internet- und Telefonapotheken von derzeit $1 \%$ auf $8 \%$ bis 2010 steigen wird (Ziegenbein, 2007, S. 4), nicht realisiert worden: Selbst in den USA, wo es große ländliche Gebiete gibt, die Preissensibilität der Nachfrager wegen des geringeren Versicherungsschutzes deutlich ausgeprägter ist, die Preise für innovative Arzneimittel wesentlich höher sind und eine Preisregulierung weitgehend fehlt, haben Versandapotheken bestenfalls einen Marktanteil von $15 \%$ erreichen können (BVDVA, 2007, S. 1). Auf jeden Fall muss die Regulierung dafür Rechnung tragen, dass nicht die Beratungsaufgabe von den öffentlichen lokalen Apotheken zu übernehmen ist, während die Versandapotheken „Rosinen picken" dürfen.

\subsubsection{Zusätzliche Lockerung der Bestimmungen zum Fremdbesitz von Apotheken}

Weitaus größere Folgen als eine Aufweichung der Mehrbesitzregelungen hat eine Lockerung der Bestimmungen zum Fremdbesitz von Apotheken. Eine solche Deregulierung würde es Einzelhändlern, die bereits Erfahrung mit dem Betrieb eines Filialsystems haben, ermöglichen, in den Markt für Arzneimitteldistribution einzusteigen.

Die neuen Marktteilnehmer könnten neben der Managementerfahrung auch auf erheblich mehr Kapital zurückgreifen, um eine Vielzahl neuer Filialen zu eröffnen oder bestehende Apotheken zu übernehmen. Allerdings wird dies auch für Einzel- und Großhandelskonzerne nur dann interessant sein, wenn sie die Apotheken in ihre bestehenden Filialnetze integrieren können oder Vorteile aus einer vertikalen Integration erzielen können.

Der Einstieg in den Apothekenmarkt ist für neue Akteure mit erheblichen Kosten zur Markterkundung verbunden. Der Erwerb bestehender Strukturen könnte hierzu eine attraktive Alternative sein.

\subsection{Wirkung einer Preisobergrenze für verschreibungs- pflichtige Medikamente bei gleichzeitiger Reduktion der Versicherungsdeckung}

Bei einer zusätzlichen Abschaffung der Vollversicherung für Arzneimittelkosten bei gleichzeitiger Definition der Arzneimittelpreise zu (durch die Apotheke) „unterbietbaren “ Preisobergrenzen wäre längerfristig von einem Margenverfall in der Arzneimitteldistribution auszugehen. ${ }^{7}$ Große Unternehmen dürften mittels ihrer Marktmacht den letztendlich für den Verbraucher wahrnehmbaren Preis drücken, die Verbraucher sollten dies zudem selber spüren, d.h. zahlen müssen.

In der Literatur existiert hier eine Vielzahl von geeigneten Vorschlägen den gewünschten Preiswettbewerb gesetzlich auszugestalten (z.B. durch eine stetige und nicht begrenzte prozentuale Zuzahlung oder einen von der Apotheke frei wählbaren Vertriebszuschlag). Experten gehen von möglichen Preissenkungen im Bereich von etwa 15\% aus. ${ }^{8}$ Die Zahl der Marktteilnehmer würde in der Folge sinken. Der beschriebene Margenverfall wäre dabei für Apotheken, die bereits unter den heutigen Gegebenheiten nur geringe Gewinne erzielen, kritisch. Große Auswirkungen hätte der Wettbewerb für Apotheken in dichter besiedelten Regionen mit einer hohen Apothekendichte, wo es den Patienten leicht möglich wäre, Preise zu vergleichen und zum günstigsten Anbieter zu wechseln.

Bedingt durch den höheren Wettbewerbsdruck könnten Apotheker nun eher gezwungen sein, ihre Apotheken zu verkaufen. Neue Marktteilnehmer könnten nicht nur Filialen eröffnen, sondern hätten durch den Preiswettbewerb ein Mittel, um Kunden noch wirksamer anzulocken. Käme es zu einem „echten“ Wettbewerb, wäre zunächst eine Steigerung der Apothekenanzahl zu erwarten. Im Zeitverlauf würde die Apothekendichte jedoch sinken. Betroffen von der Wettbewerbsverschärfung wären in diesem Fall, neben den gut gehenden Apotheken in dicht besiedelten Regionen, auch solche in weniger dicht besiedelten Regionen, die besonders ,profitable' Patienten versorgen.

Zieht man zusätzlich eine vereinfachte Zulassung von Versandapotheken in Betracht, so erhalten auch diese durch die liberalisierte Preisbildung ein wirksames Mittel, um Kunden anzuziehen. Da sie in diesem Szenario Kostenvorteile an die Kunden weitergeben können, ist von einem größeren Markterfolg dieser Anbieter als bislang auszugehen. Der Blick auf den liberalen US-amerikanischen Markt zeigt jedoch auch, dass nicht zu erwarten ist, dass diese Vertriebform auf absehbare Zeit zum dominierenden Kanal heranwächst. Insbesondere wären Versandapotheken jedoch für Patienten mit besonders teueren Medikamenten interessant.

\subsection{Folgen der Deregulierungsmaßnahmen}

Zusammenfassend kommt einer Liberalisierung der Preise und vor allem der Fremdbesitzregelung eine ganz besonders wichtige Bedeutung zu. Von den Effekten wären insbesondere Apotheken in dicht besiedelten Regionen 
betroffen. Wenn jedoch der Fremdbesitz von Apotheken nicht zugelassen wird und es bei Festpreisen bleibt, ist zu erwarten, dass sich die Struktur der Arzneimitteldistribution nur langsam ändert. Die Tabelle 2 fasst diese Überlegungen zusammen:

\section{Ausblick und Zusammenfassung}

Es hat sich gezeigt, dass eine Reihe von Regulierungen durchaus ihre Begründung haben, jedoch andere Teile des Regulierungsbündels erhebliche volkswirtschaftliche

Tabelle 2: Zusammenfassung der Folgen einzelner Deregulierungsmaßnahmen

\begin{tabular}{|c|c|c|c|c|c|c|c|c|c|}
\hline \multirow[b]{2}{*}{ Preise } & \multicolumn{9}{|c|}{ Kombination einzelner Maßnahmen der Regulierung } \\
\hline & Fest & Fest & Fest & Fest & Dereg & Dereg & Dereg & Dereg & Dereg \\
\hline $\begin{array}{l}\text { Vollversiche- } \\
\text { rung }\end{array}$ & ja & ja & ja & ja & nein & nein & nein & nein & nein \\
\hline Mehrbesitz & keine $\ddot{A}$ & Dereg & Dereg & Dereg & keine $\ddot{A}$ & Dereg & Dereg & Dereg & Dereg \\
\hline Fremdbesitz & keine $\ddot{A}$ & keine $\ddot{A}$ & Dereg & Dereg & keine $\ddot{A}$ & Keine $\ddot{A}$ & Dereg & Dereg & Dereg \\
\hline $\begin{array}{l}\text { Abgeschlossen- } \\
\text { heit/EHA }\end{array}$ & keine $\ddot{A}$ & keine $\ddot{A}$ & keine $\ddot{A}$ & Dereg & keine $\ddot{A}$ & Keine $\ddot{A}$ & keine $\ddot{A}$ & Dereg & Dereg \\
\hline Versand & keine $\ddot{A}$ & keine $\ddot{A}$ & keine $\ddot{A}$ & keine $\ddot{A}$ & keine $\ddot{A}$ & Keine $\ddot{A}$ & keine $\ddot{A}$ & keine $\ddot{A}$ & Dereg \\
\hline \multicolumn{10}{|c|}{ Folgen: } \\
\hline $\begin{array}{l}\text { Allgemeine } \\
\text { Einschätzung }\end{array}$ & $\begin{array}{l}\text { Status } \\
\text { quo }\end{array}$ & $\begin{array}{c}\text { Apothe- } \\
\text { ker eröff- } \\
\text { nen sehr } \\
\text { langsam } \\
\text { neue } \\
\text { Filialen }\end{array}$ & $\begin{array}{c}\text { Neue } \\
\text { Spieler } \\
\text { eröffnen } \\
\text { neue } \\
\text { Apothe- } \\
\text { ken }\end{array}$ & $\begin{array}{c}\text { Neue } \\
\text { Spieler } \\
\text { nutzen } \\
\text { beste- } \\
\text { hende } \\
\text { Läden, } \\
\text { um in } \\
\text { den } \\
\text { Markt } \\
\text { einzutre- } \\
\text { ten }\end{array}$ & \begin{tabular}{|c|} 
lang- \\
samer \\
Preiswett- \\
bewerb \\
der beste- \\
henden \\
Einheiten \\
\end{tabular} & $\begin{array}{c}\text { Ausdeh- } \\
\text { nung von } \\
\text { Apo- } \\
\text { theker- } \\
\text { Ketten, } \\
\text { Preiswett- } \\
\text { bewerb }\end{array}$ & $\begin{array}{l}\text { Ausdeh- } \\
\text { nung von } \\
\text { "neuen" } \\
\text { Ketten, } \\
\text { mehr } \\
\text { Preiswett- } \\
\text { bewerb }\end{array}$ & $\begin{array}{l}\text { Ausdeh- } \\
\text { nung von } \\
\text { Ketten } \\
\text { in beste- } \\
\text { henden } \\
\text { Geschäften, } \\
\text { noch mehr } \\
\text { Preiswett- } \\
\text { bewerb }\end{array}$ & $\begin{array}{l}\text { Ausdeh- } \\
\text { nung von } \\
\text { Ketten } \\
\text { in beste- } \\
\text { henden } \\
\text { Geschäften, } \\
\text { noch mehr } \\
\text { Preiswettbe- } \\
\text { werb und } \\
\text { Wettbewerb } \\
\text { durch Ver- } \\
\text { sand } \\
\end{array}$ \\
\hline Dynamik & & gering & groß & $\begin{array}{l}\text { sehr } \\
\text { groß }\end{array}$ & mittel & mittel & groß & sehr groß & sehr groß \\
\hline $\begin{array}{l}\text { Veränderung } \\
\text { der Anzahl der } \\
\text { Marktteilneh- } \\
\text { mer }\end{array}$ & & + & ++ & +++ & - & $\begin{array}{c}\text { zunächst } \\
+ \text { danach } \\
-\end{array}$ & $\begin{array}{c}\text { zunächst } \\
++, \\
\text { danach -- }\end{array}$ & $\begin{array}{c}\text { zunächst } \\
++, \\
\text { danach -- }\end{array}$ & $\begin{array}{c}\text { zunächst } \\
+++, \\
\text { danach --- }\end{array}$ \\
\hline $\begin{array}{l}\text { Apotheken in } \\
\text { dicht besiedel- } \\
\text { ten Regionen }\end{array}$ & & $\begin{array}{l}\text { kaum } \\
\text { betroffen }\end{array}$ & betroffen & \begin{tabular}{|c|} 
beson- \\
ders \\
betroffen \\
\end{tabular} & betroffen & betroffen & $\begin{array}{c}\text { sehr } \\
\text { betroffen }\end{array}$ & $\begin{array}{c}\text { sehr } \\
\text { betroffen }\end{array}$ & $\begin{array}{c}\text { sehr } \\
\text { betroffen }\end{array}$ \\
\hline $\begin{array}{l}\text { Apotheken in } \\
\text { weniger dicht } \\
\text { besiedelten } \\
\text { Regionen }\end{array}$ & & nein & nein & betroffen & $\begin{array}{l}\text { weniger } \\
\text { betroffen }\end{array}$ & $\begin{array}{l}\text { weniger } \\
\text { betroffen }\end{array}$ & betroffen & betroffen & betroffen \\
\hline $\begin{array}{l}\text { Apotheken } \\
\text { mit besonders } \\
\text { profitablen } \\
\text { Patienten }\end{array}$ & & nein & nein & nein & \begin{tabular}{|} 
in dichter \\
besie- \\
delten \\
Regionen
\end{tabular} & $\begin{array}{l}\text { weniger } \\
\text { betroffen }\end{array}$ & betroffen & betroffen & $\begin{array}{c}\text { sehr } \\
\text { betroffen }\end{array}$ \\
\hline $\begin{array}{l}\text { Nutzung der } \\
\text { Effizienz- } \\
\text { reserven }\end{array}$ & & $\begin{array}{l}\text { Mehr } \\
\text { Apothe- } \\
\text { ken }\end{array}$ & $\begin{array}{l}\text { Mehr } \\
\text { Apothe- } \\
\text { ken }\end{array}$ & $\begin{array}{l}\text { Mehr } \\
\text { Apothe- } \\
\text { ken }\end{array}$ & $\begin{array}{l}\text { Mehr } \\
\text { Apothe- } \\
\text { ken und } \\
\text { Kosten- } \\
\text { senkung }\end{array}$ & $\begin{array}{l}\text { Mehr } \\
\text { Apothe- } \\
\text { ken und } \\
\text { Kosten- } \\
\text { senkung }\end{array}$ & $\begin{array}{l}\text { Mehr } \\
\text { Apothe- } \\
\text { ken und } \\
\text { Kosten- } \\
\text { senkung }\end{array}$ & $\begin{array}{l}\text { Drogerie- } \\
\text { Apotheken } \\
\text { und Kosten- } \\
\text { senkung }\end{array}$ & $\begin{array}{l}\text { Drogerie- } \\
\text { Apotheken } \\
\text { und Kosten- } \\
\text { senkung }\end{array}$ \\
\hline
\end{tabular}


Kosten verursachen. Die staatlichen (Zulassungs-)Regulierungen können immer nur die Inputqualität, nicht jedoch die Prozess- oder Ergebnisqualität beeinflussen. Letztere lassen sich jedoch durch einen dynamischen Wettbewerb optimieren, d.h. durch Wettbewerbsfreiheit - unter Wahrung überwachter Minimalstandards.

Eine besondere Aufmerksamkeit widmet diese Untersuchung den Fremd- und Mehrbesitzregelungen, den Regelungen des Geschäftsbetriebs und der Preisregulierung. Dabei wird deutlich, dass die Wirkungen einzelner Deregulierungen als eher gering einzuschätzen sind, während mehrere Deregulierungsmaßnahmen gleichzeitig zu erheblichen Veränderungen führen können. So wird von einer alleinigen Aufhebung der Mehrbesitzregelung nur ein geringer Effekt auf die Marktstruktur, die Effizienz sowie die Qualität der Arzneimitteldistribution und damit auf die Situation der bestehenden Apotheken erwartet. Die Lockerung der Fremdbesitzbestimmungen hätte hingegen eine größere Wirkung und würde die Effizienz der Arzneimitteldistribution sowie die durchschnittliche Beratungs- und Servicequalität erhöhen. Fällt gleichzeitig die Preisregulierung und sinkt der Umfang des GKV-Versicherungsschutzes, beispielsweise durch erhöhte Selbstbeteiligungen der Patienten, so werden die traditionellen Apotheken unter einen erheblichen Wettbewerbsdruck geraten.

Es kann festgehalten werden, dass die bisherigen Beschränkungen der Bildung einer optimalen Apothekenbetriebsgröße entgegenstehen und deshalb aus ökonomischer Perspektive - entgegen des aktuellen Urteils des EuGH von Mitte Mai 2009 - mit dem Ziel einer gesteigerten Effizienz in der Arzneimittelversorgung aufgehoben werden sollten. Die Sicherstellung der ordnungsgemäßen Arzneimittelversorgung wäre auch bei weniger regulatorischen Eingriffen in den Apothekenmarkt gewährleistet.

Als Marktergebnis im Falle einer Deregulierung wäre zu erwarten, dass Apothekengroßbetriebe einen signifikanten Marktanteil erreichen und ineffiziente Apotheken größtenteils aus dem Markt gedrängt oder an Apothekengroßbetreiber verkauft werden, wodurch die Apothekenzahl (nach kurzzeitigem Anstieg) langfristig absinken könnte. Ob und mit welcher Geschwindigkeit sich die Zahl der Apotheken von derzeit etwa 21.600 verringern wird, kann nur schwer vorhergesagt werden. Aber selbst wenn die Zahl sich um ein Viertel reduzieren würde, wäre eine Unterversorgung ländlicher Gebiete noch nicht zu befürchten. Gerade in diesen Bereichen geringeren Wettbewerbs gäbe es - bei entsprechender Deregulierung und damit Spielräumen für den „Unternehmer Apotheker“ - einen Anreiz sich niederzulassen. Es ist zu erwarten, dass die verbleibenden inhabergeführten und damit marktfähigen Anbietern auf der einen Seite und solche, die in Ketten organisiert sind auf der anderen Seite, um die Gunst des Kunden konkurrieren werden.

\section{Fußnoten:}

1 Für weitere Informationen zu Regulierungsbegründungen - vor allem im Gesundheitssektor - vgl. Schulenburg/ Greiner, 2007, S. 106ff.

2 Das Bundesverfassungsgericht hat das Mehrbesitzverbot mit den Eigenarten von Arzneimitteln (Gefährlichkeit) und denjenigen des Apothekerberufs (freier Beruf) begründet. Durch eine Zulassung von Fremd- und Mehrbesitz wäre der Charakter des Apothekers als freier Beruf gefährdet. Siehe hierzu Schöffski, 1995, S. 111.

3 Dies ließ sich in der Realität auch im Anschluss an die umfassende Deregulierung des Apothekenmarktes in Norwegen beobachten.

4 Siehe hierzu auch Schulenburg, 1987 oder Schulenburg, 1993, der das Hotelling-Modell zur Analyse von Märkten mit Nachfragerpräferenzunterschieden verwendet.

5 Anders als der deutsche Strommarkt, in welchem enorme Skaleneffekte ('economies of scale') aufgrund der vorhandenen Leistungsnetze existieren. Nach Deregulierung entstand hier aus diesem natürlichen Monopol ein starres Oligopol ohne echten Wettbewerb. Auf dem Apothekenmarkt ist das genaue Gegenteil der Fall: Der Markt kommt von einem Polypol und entwickelt sich erst hin zu einer stärkeren Konzentration.

6 Siehe hierzu auch Monopolkommission, 2006, S. 416ff., Ziffer 1157ff.; S. 420, Ziffer 1178f; wobei nicht alle Einschätzungen der Kommission z.B. zu dem Betrieb von Apotheken in Drogerien - geteilt werden.

7 Im Falle eines perfekt funktionierenden Wettbewerbs wären langfristig sogar die definierten Preisobergrenzen überflüssig.

8 Siehe hierzu die verschiedenen Konzepte, beispielsweise SVR-Gesundheit, 2002, S. 70f., 279; Monopolkommission, 2006, S. 418f., Ziffer 1166ff. oder Glaeske/ Klauber/ Lankers/ Selke, 2003, S. 45ff.

\section{Literatur:}

Arzneimittelpreisverordnung (AMPreisV): Vom 14. November 1980 (BGBI. I S. 2147), zuletzt geändert durch Artikel 32 und 33 des Gesetzes vom 26. März (BGBI. I S. 378).

Bertrand, J. (1883): Review of Walras` Théorie Mathématique de la Richesse Sociale and of Cournot's Recherches sur les Principes Mathématiques de la Théorie des Richesses, Journal des Savants, 499-508.

Blohorn, H. (2006): Apothekenmarketing, OTC-Tools, 9. Jg., Heft 4, 10-11.

Bühler, S./ Jäger, F. (2002): Einführung in die Industrieökonomik, Springer, Berlin.

Bundes-Apothekerordnung (BApO): In der Fassung der Bekanntmachung vom 19. Juli 1989 (BGBI. I S. 1478, 1842), zuletzt geändert durch Artikel 33 der Verordnung vom 31. Oktober 2006 (BGBI. I S. 2407).

Bundesverband deutscher Versandapotheken, BVDVA (2007): ArzneiVersandhandel: Status Quo, Stand 1.Oktober 2007, URL: http://www. bvdva.de/fileadmin/content/pdf/Daten_und_Fakten_zum_Versandhandel_okt_07.pdf [Stand 30.06.2009].

Bundesvereinigung Deutscher Apothekerverbände, ABDA (2009): Die Apotheke - Zahlen, Daten, Fakten 2008, URL: http://www.abda.de/ fileadmin/downloads/ZDF/72dpi_ABDA_ZDF-Broschuere_100x210.pdf [Stand 03.07.2009].

Cournot, A. (1838): Recherches sur les Principes Mathématiques de la Théorie des Richesses, Paris.

Gesetz über das Apothekenwesen (ApoG): In der Fassung der Bekanntmachung vom 15. Oktober 1980 (BGBI. I S. 1993), zuletzt geändert durch Artikel 36 des Gesetzes vom 26. März 2007 (BGBI. I S. 378 Änderung durch Art. 3 G v. 02.12.2007 I 2686 (Nr. 60) textlich nachgewiesen, dokumentarisch noch nicht bearbeitet).

Glaeske, G./Klauber, J./Lankers, C.H.R./ Selke, G.W. (2003): Stärkung des Wettbewerbs in der Arzneimittelversorgung zur Steigerung von Konsumentennutzen, Effizienz und Qualität: Gutachten im Auftrag des Bundesministeriums für Gesundheit und Soziale Sicherung (BMGS), Bonn, URL: http://www.gesundheitspolitik.net/01_gesundheitssystem/ reformkonzepte/arzneimittelversorgung/Gutachten-BMGS_20030416. pdf [Stand 30.06.2009].

Hotelling, H. (1929): Stability in Competition, Economic Journal, Vol. 39, 41-57.

Kierndorfer, A. (2007): Der Gewinn liegt im Einkauf, Apotheke + Marketing, 16. Jg., 31-37.

Lindstädt, H./Hauser, R. (2004): Strategische Wirkungsbereiche von Unternehmen, Gabler, Wiesbaden.

Monopolkommission (2006): Mehr Wettbewerb auch im Dienstleitungssektor, Sechzehntes Hauptgutachten der Monopolkommission 2004/05, Nomos Verlagsgesellschaft, Baden-Baden. 


\section{THEMA}

Niestraht, U. (1983): Nachfragemacht des Handelns: Begriff, Theorie und Operationalisierung, Europäische Hochschulzeitschriften, Reihe 5, Volks- und Betriebswirtschaft, Bd. 437, Frankfurt.

Pfähler, W./ Wiese, H. (2005): Unternehmensstrategien im Wettbewerb Eine spieltheoretische Analyse, Springer, 2. Auflage, Berlin.

Sachverständigenrat für die Konzentrierte Aktion im Gesundheitswesen, SVR Gesundheit (2002): Zur Steigerung von Effizienz und Effektivität der Arzneimittelversorgung in der gesetzlichen Krankenversicherung (GKV), Addendum zum Gutachten 2000/2001: Bedarfsgerechtigkeit und Wirtschaftlichkeit, Nomos Verlagsgesellschaft, Baden-Baden.

Schulenburg, J.-M. Graf von der (1987): Marktgeschehen bei unvollständigen Nachfragerinformationen - die Auswirkungen von Anbieterwech selkosten und Informationskosten in dynamischen Marktprozessen, Zeitschrift für Betriebswirtschaft, Bd. 57, 89-109.

Schulenburg, J.-M. Graf von der (1993): Marktprozess und Marktstruktu bei unvollständigen Informationen, Zeitschrift für Wirtschafts- und Sozialwissenschaften, Vol. 113, 509-555.

Schulenburg, J.-M. Graf von der/ Greiner, W. (2007): Gesundheitsökonomik, Mohr Siebeck, 2. Auflage, Tübingen.

Schumann, J./ Meyer, U./ Ströbele, W. (2007): Grundzüge der mikroökonomischen Theorie, 8. Auflage, Springer, Berlin.
Schöffski, O. (1995): Die Regulierung des deutschen Apothekenwesens Eine ökonomische Analyse, Nomos Verlagsgesellschaft, Baden-Baden Sweezy, P.M. (1939): Demand under Conditions of Oligopoly, Journal of Political Economy, Vol. 47, 563-573.

Varian, H. (2004): Grundzüge der Mikroökonomik, Oldenbourg, 6. Auflage, München

Verordnung über den Betrieb von Apotheken (ApBetrO): In der Fassung der Bekanntmachung vom 26. September 1995 (BGBI. I S. 1195), zuletzt geändert durch Artikel 4 des Gesetzes vom 20. Juli 2007 (BGBI. I S. 1574).

Wied-Nebbeling, S. (1997): Markt- und Preistheorie, Springer, 3. Auflage, Berlin.

Woeckener, B. (2006): Einführung in die Mikroökonomik, Springer, Berlin ZEIT (2006): Billige Pillen dank EU, 33/2006 vom 10.08.2006, URL: http:// www.zeit.de/online/2006/33/presseschau-apotheken-docmorris [Stand 30.06.2009].

Ziegenbein, R. (2007): Der Apotheker als Kaufmann - Das überfachliche Kompetenzprofil des erfolgreichen Pharmazeuten, International School of Management gGmbH, URL: http://www.bvdva.de/fileadmin/ content/pdf/aktuelles/ umfragen/Studie_Qualifizierungsbedarf_V01-1. pdf [Stand 19.03.2008].

\section{Die Entdeckung des mündigen Patienten}

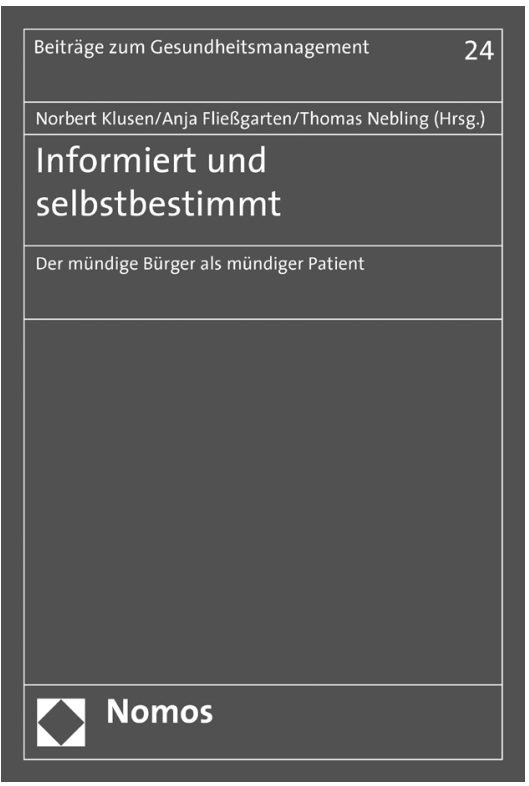

Informiert und selbstbestimmt

Der mündige Bürger als mündiger Patient

Herausgegeben von Prof. Dr. Norbert Klusen, Anja Fließgarten, M.A. und Dipl.-Gesundheitsökonom Thomas Nebling

2009, 425 S., brosch., 54,- $€$ ISBN 978-3-8329-4474-2

(Beiträge zum Gesundheitsmanagement, Bd. 24)

Wie können mündige Patienten die Gesundheitsversorgung verbessern? Welche Handlungsstrategien lassen sich hieraus für Leistungserbringer, Krankenkassen und Gesundheitspolitik ableiten? Für alle, die an der Organisation der Gesundheitsversorgung unmittelbar oder mittelbar beteiligt sind, werden diese Fragen von Autoren verschiedener Disziplinen diskutiert.

"Der Band gibt ein anregendes und gut informierendes Bild über vielfältige Aktivitäten, Strategien und Ansätze, deren Ziel es ist, das Selbstbestimmungsrecht des Patienten in der Praxis zu stärken und das Versorgungssystem patientenorientiert zu gestalten."

Prof. Dr. Robert Francke, G+S 3-4/og
Bitte bestellen Sie im Buchhandel oder versandkostenfrei unter $\downarrow$ www.nomos-shop.de
Nomos 